Results A total of 1894 adults participated in the study, of which $57.4 \%$ were women. The prevalences of overweight and obesity were $58 \%$ and $23.8 \%$, respectively. About $40 \%$ of the participants reported they perceived their weight as normal. However, when selfperceived body weight was indirectly assessed, $63.3 \%$ reported a desire to weigh less. Among the overweight and obese participants, $61 \%$ and $86.4 \%$ perceived themselves as fat or very fat, respectively, and reported a desire to weigh less.

Conclusion In this sample with high prevalences of overweight and obesity, most participants perceived their excess weight and reported a desire to weigh less. This seems an ideal setting for the implementation of interventions targeting weight loss and promoting healthy habits.

\section{SP1-57 EUROPEAN BONE TUMOUR OUTCOME STUDY (EBTOS): OVERVIEW OF HEALTH RELATED OUALITY OF LIFE (OOL) IN STUDY COHORT}

doi:10.1136/jech.2011.142976n.34

${ }^{1} \mathrm{M}$ Brown, ${ }^{1} \mathrm{M}$ Pearce, ${ }^{*}{ }^{1} \mathrm{~J}$ Salotti, ${ }^{2} \mathrm{~A}$ Craft, ${ }^{3} \mathrm{~J}$ Hale. ${ }^{1}$ Institute of Health \& Society, Newcastle University, Newcastle Upon Tyne, UK; ${ }^{2}$ Sir James Spence Institute, Royal Victoria Infirmary, Newcastle Upon Tyne, UK; ${ }^{3}$ Paediatric Oncology, Royal Victoria Infirmary, Newcastle Upon Tyne, UK

Introduction Survivors of bone tumours diagnosed in childhood or early adulthood are reported to have more impairment of health related QOL than most other cancer survivor groups. Reports of the relationship between QOL and local treatment strategy have been inconsistent. To clarify this issue a European cohort of bone tumour survivors was identified. This report forms a baseline for further analyses.

Methods Osteosarcoma and Ewing's sarcoma survivors were $>5$ years from diagnosis, $<40$ yrs at diagnosis and $>16$ yrs at time of survey. 1145 eligible survivors were mailed a questionnaire which included socio-demographic data, recall of treatment, SF36, Rosenberg Self Esteem Scale, EORTC Body Image \& Sexuality modules and TESS to assess physical function. Statistical analysis was with $\chi^{2}$ and t test using Stata software.

Results 714 (62\%) survivors returned questionnaires (253 UK, 31 Netherlands, 430 DE). Median age at diagnosis was 15 yrs (1-38) and at survey was $26 y$ ys (16-52). Time since diagnosis was 5-20 yrs (median 11). Survivors had: Ewing's sarcoma (319), osteosarcoma (395), upper extremity site (107), lower (535), axial (72), amputation (161), rotationplasty (51), limb salvage (502). Most were single and childless, only $6 \%$ were unemployed while $64 \%$ felt that job opportunities were affected. SF36 scores showed impaired physical (UK and DE) and mental health (DE) compared to population norms, but enhanced self esteem. There were national differences in sub-scale scores.

Conclusions This overview of the EBTOS cohort confirms areas of impaired QOL balanced by enhanced self-esteem, while also demonstrating variability between the national groups.

\section{SP1-58 MALNUTRITION, QUALITY OF LIFE AND CANCER: ASSOCIATION BETWEEN DIFFERENT NUTRITIONAL PARAMETERS}

doi:10.1136/jech.2011.142976n.35

${ }^{1} \mathrm{~S}$ P Orlandi, ${ }^{1} \mathrm{M}$ C F Assunção, ${ }^{2} \mathrm{C}$ A Pastore, ${ }^{2} \mathrm{M}$ C Gonzalez. ${ }^{1}$ Post Graduation in Epidemiology, Federal University of Pelotas, Pelotas, Rio Grande do Sul, Brazil, ${ }^{2}$ Post Graduation in Health and Behaviour, Catholic University of Pelotas, Pelotas, Rio Grande do Sul, Brazil

Introduction Weight loss is a frequent complication in patients with cancer, and it is present in almost $85 \%$ of patients in some specific kinds of tumour. Malnutrition, decreased functional capacity and quality of life (QOL) contribute to an increased morbidity and mortality in these patients.

Objectives To evaluate the association among nutritional status, quality of life, index of fat free mass (FFMI) and functional capacity in patients undergoing chemotherapy.

Methods A prospective study was conducted in patients before the first chemotherapy course in the Hospital of Federal University of Pelotas, Brazil. Nutritional status was determined by PatientGenerated Subjective Global Assessment and quality of life by the European Organization for Research and Treatment of Cancer Quality of Life Questionnaire. Bioelectrical impedance analysis and hand grip strength (HGS) were performed to evaluate FFMI and functional capacity, respectively.

Results 75 patients were evaluated, $74.7 \%$ had cancer of the digestive system. Only $12.8 \%$ of the patients were classified as well nourished. HGS median was $26 \mathrm{~kg}$ (IOR:18; $32 \mathrm{~kg}$ ) FFMI median was $17.5 \mathrm{~kg} / \mathrm{m}^{2}$ (IOR:16.1; $18.8 \mathrm{~kg} / \mathrm{m}^{2}$ ). Severely malnourished patients had a worse functional capacity $(p=0.01)$; lower FFMI $(p=0.005)$ and lower general QOF scores $(p=0.03)$ than the nourished ones. A significant positive correlation was found between IFFM and HGS $(r=0.51 ; p<0.001)$ and significant negative correlations between functional capacity and general $\mathrm{OOL}(\mathrm{r}=-0.48$; $\mathrm{p}<0.001)$ and HGS $(\mathrm{r}=-0.44 ; \mathrm{p}<0.001)$.

Conclusions Malnutrition is a determining factor in the prognosis and QOL in these patients and it can be assessed by different parameters such as body composition, subjective evaluations or changes in functional capacity.

\section{SP1-59 DEPRESSION AMONG ELDERLY PEOPLE IN AN OLD HOME OF DHAKA CITY}

doi:10.1136/jech.2011.142976n.36

${ }^{1} \mathrm{M}$ Moula, ${ }^{1} \mathrm{~S}$ J Mumu, * ${ }^{2} \mathrm{M}$ S Flora. ${ }^{1}$ Bangladesh Institute of Health Sciences (BIHS), Dhaka, Bangladesh; ${ }^{2}$ National Institute of Preventive and Social Medicine (NIPSOM), Dhaka, Bangladesh

Introduction With a rapidly ageing society, geriatric depression is emerging as important public health concern. The untreated depressed elderly patients have significant clinical and social implications as these disorders decrease an individual's quality of life and increase dependence on others. The present study aimed to assess the level of depression and its associated factors among the elderly (60 years old and above) in an old home.

Methods Under a cross sectional design 107 respondents aged $>60$ years were selected purposively from an Old Home of Dhaka city, Bangladesh. A 30-item Geriatric Depression Scale (GDS) questionnaire was used to assess the level of depression with a score $\leq 9$ as normal or non-depressive, $10-19$ as mild and 20-30 as severely depressed.

Results Mean age of study participants was $69.4 \pm 5.1$ years and $63 \%$ were male, $46.7 \%$ illiterate, $61.7 \%$ from rural area. About half of elderly respondents were having some degree of depression of whom $8.4 \%$ were severely depressed. Depression was more common in females $(60 \%)$ than males $(40 \%)$. Depression was significantly associated with nuclear family $(p=0.009)$, low family income $(p=0.001)$, female gender $(p=0.048)$, chronic illness $(p=0.042)$ and widow or widowers $(p=0.036)$. No influence of educational status and living area was found.

Conclusion This study showed that proportion of depression was high among elderly and depression was significantly associated with nuclear family, female gender, chronic illness, widow/ widower and low family income. Along with other illness depression should also be one of the key areas of concern for the elderly population. 